\title{
COUNTABLY GENERATED FAMILIES
}

\author{
R. DANIEL MAULDIN
}

\begin{abstract}
This paper discusses some interrelationships between various statements involving sets generated by rectangles, universal spaces, and realvalued measures on the continuum. Borel sets on ordinal spaces are also discussed.
\end{abstract}

Definition. Let $E$ denote a set. If $A$ is a subset of $E^{2}=E \times E$, let $A_{x}=\{y \mid(x, y) \in A\}$, for each $x$ in $E$ and let $A^{y}=\{x \mid(x, y) \in A\}$, for each $y$ in $E$. Let $R$ be the family of all sets of the form $A \times B$ in $E^{2}$.

If $G$ is a subset of $2^{E}$, let $G_{0}$ be $G$ and for each ordinal $\alpha$, let $G_{\alpha}$ be the family of all countable unions (intersections) of sets in $\cup_{\gamma<\alpha} G_{\gamma^{\prime}}$ if $\alpha$ is odd (even). Limit ordinals will be considered even. Of course, $G_{\omega_{1}}$ is the smallest family including $G$ which is closed under countable unions and intersections; $G_{\omega_{1}}$ is the Borel lattice generated by $G$. It can be checked that if for each $A \in G$, $A^{\prime} \in G_{\omega_{1}}$, then $G_{\omega_{1}}$ is closed under complements and $G_{\omega_{1}}$ is then the $\sigma$-algebra or Borel algebra generated by $G$.

In [1], a study is made of the Borel lattice (algebra) generated by the family $R$ and a number of the results stated in that paper will be used here. In particular, if $|E|>c$, then the main diagonal in $E \times E$ is not in $R_{\omega_{1}}$. If $|E| \leqslant \omega_{1}$, then $2^{E^{2}}=R_{\omega_{1}}=R_{\sigma \delta}$. (It should be noted that throughout this paper, the Axiom of Choice is assumed and cardinals are regarded as initial ordinals.)

Kunen [4] investigated the family $R_{\omega_{1}}$ when $|E| \leqslant c$. He showed that Martin's Axiom implies $R_{\sigma \delta}=2^{E^{2}}$, if $|E|^{1} \leqslant c$.

Recently, Franklin Tall and Kenneth Kunen have constructed a model of ZFC in which Martin's Axiom fails and yet $2^{E^{2}}=R_{\omega_{1}}$, if $|E| \leqslant c$. R. Mansfield [18] and B. V. Rao [19], [20] have also studied the sets generated by rectangles and have solved some of Ulam's problems with their aid.

There are a number of interesting consequences which follow from the assumption that $2^{E^{2}}=R_{\omega_{1}}$ and from the techniques which have been used in the study of families generated by $R$.

\section{Results.}

THEOREM 1. Suppose $2^{E^{2}}=R_{\sigma \delta}$, where $|E|=c$, and if $\lambda<c$, then $2^{\lambda} \leqslant c$. Then there is a subset $M$ of $[0,1]$ such that the Banach space $B_{1}(M)$ consisting of the bounded real-valued functions of Baire's class 1 defined on $M$ and under the uniform norm is universal for all Banach spaces of cardinality $c$.

Received by the editors November 15, 1974.

AMS (MOS) subject classifications (1970). Primary 04A15, 04A30; Secondary 28A05.

Key words and phrases. Rectangles, continuum hypothesis, Martin's Axiom, universal space, measurable cardinal, ray of ordinals, Borel sets. 
Proof. It is known that the condition $\lambda<c$ implies $2^{\lambda} \leqslant c$ is equivalent to $c=c^{c}$. It is known that $c=c^{c}$ implies there is a zero-dimensional compact $T_{2}$ space $U$ of weight $c$ such that if $Y$ is a compact $T_{2}$ space weight $\leqslant$ $c$, then $Y$ is a continuous image of $U$ [16], [17, p. 131]. If $X$ is a Banach space and $|X|=c$, then $X$ can be embedded in a space $C(S)$ where $S$ is a compact $T_{2}$ space and $|C(S)|=c$. But if $|C(S)| \leqq c$, then weight of $S$ is $\leqq c$. Thus, $X$ can be embedded in $C(U)$. Since $U$ has weight $c,|C(U)| \leqslant c$. Now by Theorem 4.4 of [7], there is a subset $M$ of $[0,1]$ such that $B_{1}(M)$ is universal for all Banach spaces of cardinality $c$.

Question. Does the existence of a universal Banach space of cardinality $c$ imply $c=c \mathcal{c}$ or that there is a compact $T_{2}$ space, $U$, of weight $c$ so that every compact $T_{2}$ space of weight $c$ is a continuous image of $U$ ?

There are a number of other consequences of the assumption that $R_{2}=R_{\sigma \delta}$ $=2^{E^{2}}$ by itself or together with a cardinality condition. For example, if $2^{\aleph_{1}}=2^{\aleph_{0}}$ and $R_{2}=2^{E^{2}}$, then there exists a $Q$-set [7, Theorem 4.5].

In [4], Kunen showed that $R_{\omega_{1}}=2^{E^{2}}$ implied that $|E|$ is not a real-valued measurable cardinal. As Kunen points out, his argument is a variant of the known fact that a well-ordering of the real numbers is not Lebesgue measurable [11]. We shall generalize this argument as follows:

TheOREM 2. Let $|E|=c$. For each positive integer $i$, statement $i$ implies statement $i+(1)$.

1. $2^{\boldsymbol{N}_{0}}=\boldsymbol{N}_{1}$;

2. Martin's Axiom;

3. $2^{E^{2}}=R_{2}=R_{\sigma \delta}$;

4. $2^{E^{2}}=R_{\omega_{1}}$;

5. there is a countable ordinal $\alpha$ such that if $H$ is a family of $c$ subsets of $E$, then there is a countable family $G$ of subsets of $E$ such that $H \subseteq G_{\alpha}$;

6. there is a countable ordinal $\alpha$ such that if $H$ is a family of $c$ subsets of $E$ and each member of $H$ has cardinality $<c$, then there is a countable family $G$ such that $H \subseteq G_{\alpha}$;

7. if $H$ is a family of $c$ subsets of $E$ and each member of $H$ has cardinality $<$ $c$, then there is a countable family $G$ such that $H$ is a subcollection of the Borel algebra generated by $G$;

8. if $W$ is a subset of $E^{2}$, then $W$ is the union of a subfamily $G$ of $R_{\omega_{1}}$ such that the cardinality of $G$ is not real-valued measurable;

9. if $\kappa$ is a real-valued measurable cardinal, then $\kappa>c$.

Proof. For the first three implications, see Kunen [4]. As has been mentioned, $3 \nrightarrow 2$. Clearly $3 \rightarrow 4$, and in [1] it is shown that $4 \rightarrow 5$. The question "Does $4 \rightarrow 3$ ?" was raised in [1]. Clearly $5 \rightarrow 6$.

We shall now show that $6 \rightarrow 4$.

Let $E$ be well ordered into an initial type; $E=\left\{x_{1}, x_{2}, \ldots, x_{\alpha}, \ldots \mid \alpha<c\right\}$. Let $A=\left\{\left(x_{\alpha}, x_{\gamma}\right) \mid \alpha<\gamma\right\}$ and let $B=E^{2}-A$. For each $y,\left|A^{y}\right|<c$ and for each $x,\left|B_{x}\right|<c$.

Let $Z \subseteq E^{2}$. Then $Z=(Z \cap A) \cup(Z \cap B)$. Let $H=\left\{(Z \cap A)^{y} \mid y\right.$ $\in E\}$. Each member of $H$ has cardinality $<c$. Thus, if 6 holds, there is a countable family of $G$ of subsets of $E$ and a countable ordinal $\alpha$ such that $H \subseteq G_{\alpha}$. It follows directly from Theorem 3 of [1] that $Z \cap A \in R_{\omega_{1}}$. Similarly, $Z \cap B \in R_{\omega_{1}}$. So, $6 \rightarrow 4$. 
It should be noted that this argument may be used to show that if $|T|=\omega_{1}$, then every subset of $T^{2}$ is in the family $R_{\sigma \delta}$. This fact is proven by different means by Kunen [4].

Also, it should be pointed out that if statement 5 holds, then if $H$ is a family of $c$ subsets of $E$, there is a countable family $G$ of subsets of $E$ such that $H \subseteq \cup_{\alpha<\omega_{1}} G_{\alpha}$. This problem was raised by Ulam and Rothberger [9], [14].

Clearly, $6 \rightarrow 7$. It is unknown to the author whether $7 \rightarrow 6$.

We shall now show that $7 \rightarrow 8$.

Let $A$ and $B$ be the sets described above. Let $W \subseteq E^{2}$ and let $H$ $=\left\{(A \cap W)^{y} \mid y \in E\right\}$. Clearly, each member of $H$ has cardinality $<c$ and $|H| \leqslant c$. Thus, from 7 , there is a $G,|G| \leqslant \omega_{0}$ such that $H \subseteq \cup_{\gamma<\omega_{1}{ }_{\gamma}} G_{\text {. }}$

For each $\gamma<\omega_{1}$, let $K_{\gamma}=\left\{(x, y) \mid(x, y) \in A \cap W\right.$ and $\left.(A \cap W)^{y} \in G_{\gamma}\right\}$. It follows from Theorem 3 of [1] that $K_{\gamma} \in R_{\gamma}$.

Thus, $W \cap A=\cup_{\gamma<\omega_{1}} K_{\gamma}$. There is a similar argument for $W \cap B$ and certainly $7 \rightarrow 8$ since $\omega_{1}$ is not a real-valued measurable cardinal.

Finally, we show $8 \rightarrow 9$. We argue indirectly. Suppose $\kappa, \kappa \leqslant c$, is realvalued measurable and 8 holds. Let $\omega_{\alpha}$ be the first real-valued measurable cardinal and let $S=\left\{\gamma \mid \gamma<\omega_{\alpha}\right\}$ and let $A=\{(x, y) \mid(x, y) \in S$ $\times S$ and $x$ precedes $y$ \}.

It follows from statement 8 that there is a subfamily $G=\left\{A_{\gamma}\right\}_{\gamma<\lambda}$ of the Borel field generated by the rectangles over $S$ such that $A=\cup_{\gamma<\lambda} A_{\gamma}$ and $\lambda$ is not real-valued measurable.

Let $\mu$ be a free probability measure on $\omega_{\alpha}$ which is $\omega_{\alpha}$-additive.

For each $\gamma<\lambda, A_{\gamma}$ is $\mu \times \mu$-measurable. We calculate the measure of $A_{\gamma}$ by Fubini's theorem.

$$
\mu \times \mu\left(A_{\gamma}\right)=\int_{S \times S} \chi_{A_{\gamma}} d(\mu \times \mu)=\int_{S}\left[\int_{S} \xi_{A_{\gamma}}(x, y) d \mu(x)\right] d \mu(y) .
$$

But, for each $y, \int_{S} \xi_{A_{\gamma}}(x, y) d \mu(x)=\mu\left(A_{\gamma}^{y}\right)=0$. Thus, $(\mu \times \mu)\left(A_{\gamma}\right)=0$.

For each $\gamma<\lambda$, let $P_{\gamma}=\left\{x \mid \mu\left(\left(A_{\gamma}\right)_{x}\right)>0\right\}$. It follows from Fubini's theorem that each $P_{\gamma}$ has $\mu$-measure 0 .

However, for each $x \in S, \mu\left(A_{x}\right)>0$ and $A_{x}=\cup_{\gamma<\lambda}\left(A_{\gamma}\right)_{x}$. Thus, $\cup_{\gamma<\lambda} P_{\gamma}$ $=S$. But, since $\mu$ is $\omega_{\alpha}$-additive, $\mu(S)=0$. This contradiction completes the proof of the theorem.

REMARK 1. The theorem that $8 \rightarrow 9$ was also proven by E. Fisher in his thesis [2]. In fact, Fisher showed that no well-ordering of $\omega_{\alpha}$ is in the $\omega_{\alpha}$ algebra generated by $R$. The author was unaware of this and thanks the referee for pointing this out and for making a number of other helpful comments.

REMARK 2. In the first issue of Colloquium Mathematicum, Banach showed that the continuum hypothesis implies that there is a countable family of subsets of $I$, the unit interval, such that Lebesgue measure cannot be extended from the Lebesgue measurable sets to a $\sigma$-algebra containing these sets. The same result holds under Martin's Axiom. Any countable family $\left\{E_{n}\right\}_{n=1}^{\infty}$ such that a well-ordering of $I$ (regarded as a subset of $I \times I$ ) is in the $\sigma$-algebra generated by the rectangles $A_{n} \times A_{m}$ will suffice. The argument is the same as above, in view of the fact that Lebesgue measure is $c$-additive under Martin's Axiom [6]. 
As mentioned earlier, it is apparently unknown whether $R_{\omega_{1}}=2^{E^{2}}$ implies $R_{2}=R_{\sigma \delta}=2^{E^{2}}$. In fact, it is apparently unknown whether there is any family of sets $G$ such that $G_{\alpha}=G_{\alpha+1}$, but $G_{\beta} \neq G_{\gamma}$ for $\beta<\alpha$ and $\alpha>3$ [3]. It is known that the Baire order of compact $T_{2}$ spaces is either 0,1 or $\omega_{1}$ (here $G$ is the family of all closed $O_{\delta}$ sets) [8]. It is apparently unknown what the Borel order of a compact $T_{2}$ space may be (here $G$ is the family of sets which are the intersection of an open set and a closed set).

We now describe the Borel subsets of the ordinal spaces $[0, \alpha)$ provided with the order topology. First, in Theorem 4, the Borel subsets of $\left[0, \omega_{1}\right)$ are described. This theorem was proven by M. Bhaskara Rao and K. P. S. Bhaskara Rao [21].

THEOREM 3. Every Borel subset of $\left[0, \omega_{1}\right)$ can be expressed as the union of countable many sets, each of which is the intersection of an open set and a closed set.

Proof. Let $\Re$ be the $\sigma$-algebra of all subsets $E$ of $\left[0, \omega_{1}\right)$ such that $E$ or $E^{\prime}$ contains a closed unbounded subset of $\left[0, \omega_{1}\right)$. Clearly $\Re$ contains the open sets and the closed sets.

Suppose $E \in \Re$ and $E^{\prime}$ contains a closed unbounded set $F_{0}$. Let $\left\{V_{\alpha}\right\}_{\alpha \in A}$ be the set of all order components of the complement of $F$. Then $E \cap V_{\alpha}$ is countable: $E \cap V_{\alpha}=\left\{x_{\alpha n}\right\}_{n=1}^{\infty}$. Let $K_{n}=\left\{x_{\alpha n} \mid \alpha \in A\right\}$. For each $n, K_{n}$ is closed in $F^{\prime}$. Thus, $K_{n}=F_{n} \cap V$, where $F_{n}=\bar{K}_{n}$ and $V=F^{\prime}$ and $E$ $=\cup_{n=1}^{\infty} K_{n}$.

If $E \in \mathcal{M}$ and $E$ contains a closed unbounded set $F_{0}$, then as before $E-F_{0}=\cup_{n=1}^{\infty}\left(F_{n} \cap V\right)$ and $E=F_{0} \cup \cup_{n=1}^{\infty}\left(F_{n} \cap V\right)$ where $V=F_{0}^{\prime}$.

Thus, $)$ is the family of all Borel subsets of $\left[0, \omega_{1}\right)$ and $E$ is a Borel subset of $\left[0, \omega_{1}\right)$ if and only if $E$ or $E^{\prime}$ contains a closed unbounded set.

REMARK 3. In contrast with the classical development, the smallest family containing the closed subsets of $\left[0, \omega_{1}\right)$ which is closed under countable unions and intersections is not the Borel algebra generated by the closed sets. In fact, let $\mathfrak{H}=\{x \mid x$ is countable or $x$ contains an unbounded closed set $\}$. Then $\mathscr{K}$ contains all the closed sets, $\mathcal{K}_{\sigma}=\mathcal{K}_{\delta}=\mathcal{K}$, and yet $\mathcal{K} \neq \mathscr{K}$.

REMARK 4. It is known that the $\sigma$-algebra generated by Borel measurable rectangles in $\left[0, \omega_{1}\right) \times\left[0, \omega_{1}\right)$ does not include all Borel subsets of $\left[0, \omega_{1}\right)$ $\times\left[0, \omega_{1}\right)$. In fact, the sets $D_{1}=\{(x, y) \mid y>x\}$ and $D_{2}=\{(x, y) \mid y<x\}$ are disjoint open sets which are not measurable with respect to the outer measure induced by the gauge $g(A \times B)=\mu(A) \cdot \mu(B)$, where $\mu$ is Dieudonne's measure: $\mu(E)=1$, if $E$ contains a closed unbounded set and $\mu(E)=0$, otherwise.

We have

$$
\mu_{g}^{*}(E)=\inf \left\{\sum_{n=1}^{\infty} g\left(A_{n} \times B_{n}\right) \mid \bigcup_{n=1}^{\infty} A_{n} \times B_{n} \supset E\right\} .
$$

It follows that $\mu_{g}^{*}$ is $\{0,1\}$-valued. We show $\mu_{g}\left(D_{1}\right)=\mu_{g}\left(D_{2}\right)=1$ to show that there are nonmeasurable Borel sets. If $\mu_{g}\left(D_{1}\right)=0$, then there is a sequence $\left\{A_{n} \times B_{n}\right\}_{n=1}^{\infty}$ such that $D_{1} \subset \cup_{n=1}^{\infty}\left(A_{n} \times B_{n}\right)$ and for each $n, A_{n}$ or $B_{n}$ fails to contain a closed unbounded set. 
Let $A_{n_{1}}, A_{n_{2}}, \ldots$ be the sequence of all the $A_{n}$ 's of $\mu$-measure 0 . Let $F$ be a closed unbounded subset of $\bigcap_{i=1}^{\infty} A_{n_{i}}^{\prime}$. Let $x \in F$ and let $K=\{y \mid y>x\}$. Then $\{x\} \times K \subset D_{1}$ and $\{x\} \times K \subset \cup_{i=1}^{\infty}\left(A_{m_{i}} \times B_{m_{i}}\right)$ where $A_{m_{i}}$ 's contain unbounded closed sets. Then no $B_{m_{i}}$ contains an unbounded set and yet $K \subset \cup_{i=1}^{\infty} B_{m_{i}}$. This is a contradiction.

Note. The referee points out that $D_{1}$ and $D_{2}$ are not measurable in $\mu \times \mu$ follows immediately by Fubini's theorem, as in the proof of $8 \rightarrow 9$ in Theorem 2.

THEOREM 4. Let $\alpha$ be an ordinal. Every Borel subset of $[0, \alpha)$ can be expressed as the union of countably many sets, each of which is the intersection of an open set and a closed set.

Proof. Clearly, the theorem holds for all ordinals $\alpha, \alpha \leqslant \omega_{1}$. It is also easy to show that if the theorem holds for the ordinal $\alpha$, then it holds for $\alpha+1$.

So assume $\alpha$ is a limit ordinal and the theorem holds for all $\beta<\alpha$. We consider two cases.

Case 1. $\operatorname{cf}(\alpha)=\alpha$.

In this case, let $\mathfrak{R}=\left\{E \mid E\right.$ or $E^{\prime}$ contains a closed unbounded set and $\forall \gamma<\alpha, E \cap[0, \gamma)$ is Borel in $[0, \gamma)\}$.

$\Re$ is a $\sigma$-algebra and $\Re$ contains both the closed and the open subsets of $[0, \alpha)$.

Suppose $E \in \Re$ and $E^{\prime} \supset F_{0}, F_{0}$ a closed unbounded set. Let $\left\{I_{\gamma}\right\}_{\gamma \in \Gamma}$ be the set of order components of $[0, \alpha)-F_{0}$.

Then, $E \cap I_{\alpha}$ is Borel in $I_{\alpha}$. Thus, $E \cap I_{\alpha}=\cup_{n=1}^{\infty}\left(F_{n \alpha} \cap O_{n \alpha}\right)$, where $F_{n \alpha}$ is closed in $I_{\alpha}$ and $O_{n \alpha}$ is open in $I_{\alpha}$.

For each $n$, let $F_{n}=\cup_{\gamma \in \Gamma} F_{n \gamma}$ and let $U_{n}=\cup_{\gamma \in \Gamma} O_{n \gamma}$. It follows that $E=\cup_{\gamma}\left(E \cap I_{\gamma}\right)=\cup_{n=1}^{\infty}\left(F_{n} \cap U_{n}\right)$.

If $E \in \Re$ and $E \supset F_{0}, F_{0}$ a closed unbounded set, then $\left(E-F_{0}\right) \supset F_{0}$ and we obtain $E=F_{0} \cup \cup_{n=1}^{\infty}\left(F_{n} \cap U_{n}\right)$.

Case II. cf $(\alpha)=\tau<\alpha$.

In this case fix a set $F_{0}=\left\{\gamma_{\beta}\right\}_{\beta<\tau}$ running through $\alpha$ and such that $F_{0}$ is closed. Let $\left\{I_{\sigma}\right\}_{\sigma \in \Sigma}$ be the set of order components of $F_{0}{ }^{\prime}$. If $E$ is Borel in [0, $\alpha$ ), then $E \cap F_{0}$ is Borel in $F_{0}$ and $E \cap I_{\sigma}$ is Borel in $I_{\sigma}$. Since $\tau<\alpha, E \cap F_{0}$ $=\cup_{n=1}^{\infty}\left(\mathcal{K}_{n} \cap V_{n}\right)$, where $\mathcal{K}_{n}$ is closed in $F_{0}$ and $V_{n}$ is open in $F_{0}$. Thus, $E \cap F_{0}=\cup_{n=1}^{\infty} F_{2 n} \cap U_{2 n}, F_{2 n}$ closed in $[0, \alpha)$ and $U_{2 n}$ open in $[0, \alpha)$. For each $\sigma \in \Sigma, E \cap I_{\sigma}=\cup_{n=1}^{\infty}\left(F_{n \sigma} \cap U_{n \sigma}\right)$. Let $F_{2 n-1}=\cup_{\sigma \in \Sigma} F_{n \sigma}$ and $U_{2 n-1}$ $=\cup_{\sigma \in \Sigma} U_{n \sigma}$. It follows that $E \cap F_{0}{ }^{\prime}=\bigcup\left(F_{2 n-1} \cap U_{2 n-1}\right)$ and $E$ $=\cup_{n=1}^{\infty}\left(F_{n} \cap U_{n}\right)$. Q.E.D.

Thus, if one considers the compact $T_{2}$ space $[0, \alpha]$, it has Borel order 1 no matter what ordinal $\alpha$ is.

Problem. Does this result hold for all compact scattered $T_{2}$ spaces? Is the Borel order of the other compact $T_{2}$ spaces $\omega_{1}$ ?

In [21], it is shown that there is no nonatomic, countably additive, finite measure defined on the Borel subsets of $\left[0, \omega_{1}\right)$. We generalize this in the next theorem.

THEOREM 5. If there is no real-valued measurable cardinal $\kappa$ with $\kappa \leqslant \alpha$, then every countably additive finite measure defined on the Borel subsets of $[0, \alpha)$ is purely atomic. 
Proof. Let us assume the contrary. Let us assume that $\alpha$ is the first ordinal for which such a measure exists and that $\mu$ is a nonatomic probability measure defined on the Borel subsets of $[0, \alpha)$.

Notice that if $E$ is a Borel subset of $[0, \alpha)$ such that $E$ is Borel isomorphic to some space $[0, \beta)$, with $\beta<\alpha$, then $\mu(E)=0$. Next notice that if $F$ is a closed cofinal subset of $[0, \alpha)$, then the open set $U=F^{\prime}$ has measure zero. This can be seen as follows: First let $\varphi$ be a $1-1$ map from $[0, \beta)$, for some $\beta \leqq \alpha$, onto the set of order components of $U$. Define $\nu$ on each subset $W$ of $[0, \beta)$ by $\nu(W)=\mu(\cup\{\varphi(\gamma): \gamma \in W\})$. Then $\nu$ is a free countably additive finite measure defined on all subsets of $[0, \beta)$. Therefore $\nu([0, \beta)]=\mu(U)=0$.

Suppose $E$ is a Borel set which fails to contain a closed cofinal set. By the previous theorem, $E=\cup_{i=1}^{\infty}\left(F_{i} \cap U_{i}\right)$, where for each $i, F_{i}$ is closed and $U_{i}$ is open. For each $i$, either $F_{i}$ or $U_{i}$ fails to contain a closed cofinal set. If $U_{i}$ does not contain such a set, then $\mu\left(U_{i}\right)=0$. If $F_{i}$ fails to contain such a set, then $F_{i}$ is a subset of an open set not containing a closed cofinal set. Therefore, $\mu(E)=0$.

Finally, notice that if $B$ is a Borel set, then either $B$ or $B^{\prime}$ contains a closed cofinal subset. But, this implies that $\mu$ is purely atomic.

REMARK. It is known that every regular Borel measure on any ordinal space (or, more generally, any compact dispersed space) is concentrated on a countable set.

\section{REFERENCES}

1. R. H. Bing, W. W. Bledsoe and R. D. Mauldin, On sets generated by rectangles, Pacific J. Math. 51 (1974), 27-36.

2. E. R. Fisher, Toward integration without fear, Ph.D. Thesis, Department of Mathematics, Carnegie Mellon University, 1969.

3. K. Kolmogorov, Problème 65, Fund. Math. 25 (1935), 578.

a. K. Kunen, Inaccessibility properties of cardinals, Ph.D. Thesis, Department of Mathematics, Stanford University, August 1968.

5. Szpilrajn-Marczewski, $O$ miersalnosi i warunku Baire's, C. R. du I Congres des Math. des Pays Slaves, Varsovi 1929, p. 209.

6. D. A. Martin and R. M. Solovay, Internal Cohen extensions, Ann. Math. Logic 2 (1970), no. 2, 143-178. MR 42 \#5787.

7. R. D. Mauldin, On generalized rectangles and countably generated families, Fund. Math. (to appear).

8. Baire functions, Borel sets and ordinary function systems, Advances in Math. 12 (1973), $418-450$.

9. F. Rothberger, On families of real functions with a denumerable base, Ann. of Math. (2) 45 (1944), 397-406. MR 6, 120.

10. M. E. Rudin, A subset of the countable ordinals, Amer. Math. Monthly 64 (1957), 351. MR 19,4 .

11. W. Sierpinski, Sur les rapports entre I existence des intégrales $\int_{0}^{1} f(x, y) d x \int_{0}^{1} f(x, y) d y$ et $\int_{0}^{1} d x \int_{0}^{1} f(x, y) d y$, Fund. Math. 1 (1920), 142-147.

12. - Sur les espaces metriques universels, Fund. Math. 33 (1945), 123-136. MR 7, 420.

13. S. M. Ulam, Zur Masstheorie in der allgemeinen Mengenlehre, Fund. Math. 16 (1930), 141150.

14. Problem 74, Fund. Math. 30(1938), p. 365.

15. - Problems in modern mathematics, Science Editions, Wiley, New York, 1964. MR $43 \# 6031$.

16. A. S. Esenin-Vol'pin, On the existence of a universal bicompactum of arbitrary weight, Dokl. Akad. Nauk SSSR 68 (1949), 649-652. (Russian) MR 11, 165. 
17. W. W. Comfort and S. Negrepontis, The theory of ultrafilters, Springer-Verlag, New York, 1974.

18. R. Mansfield, The solution to one of Ulam's problems concerning analytic sets. II, Proc. Amer. Math. Soc. 26 (1970), 539—540. MR 43 \#4988.

19. B. V. Rao, Remarks on analytic sets, Fund. Math. 66 (1969/70), 237-239. MR 43 \#451. 20. - On discrete Borel spaces and projective sets, Bull. Amer. Math. Soc. 75 (1969), 614-617. MR 39 \#4014.

21. M. Bhaskara Rao and K. P. S. Bhaskara Rao, Borel o-algebra on $[0, \Omega]$, Manuscripta Math. 5 (1971), 195-198. MR 45 \#2118.

Department of Mathematics, University of Florida, Gainesville, Florida 32611 